\title{
Status and first results from the ARCA and ORCA lines of the KM3NeT experiment
}

\author{
Paolo Fermani ${ }^{1,2, a}$ and Irene Di Palma ${ }^{1,2}$ \\ ${ }^{1}$ Sapienza Università di Roma, P.le Aldo Moro 5, 00185, Roma, Italy \\ ${ }^{2}$ INFN - Sezione di Roma, P.le Aldo Moro 2, 00185, Roma, Italy
}

\begin{abstract}
KM3NeT is a network of submarine Cherenkov neutrino telescopes under construction in two different sites in the Mediterranean Sea [1]. The detector at the Italian site, close to the Sicilian coast and named ARCA, will be devoted to the detection of highenergy astrophysical neutrinos coming from sources in the Universe, while the detector at the French site, in the Toulon bay and named ORCA, will exploit atmospheric neutrinos to determine the neutrino mass hierarchy. The telescopes are an array of flexible strings anchored to the sea floor and held close to vertical by submerged buoys. The strings are instrumented with digital optical modules hosted within pressure-resistant glass spheres, each housing 313 " photomultipliers tubes and the readout electronics. The geometry of the detectors has been adapted to their physics goals. The first calibrations and results of ARCA and ORCA are presented.
\end{abstract}

\section{Introduction}

The KM3NeT experiment infrastructure is composed by two detectors: ARCA and ORCA. In ARCA, every string is $700 \mathrm{~m}$ high and has 18 multi-photomultiplier tubes (PMTs) digital optical modules (DOMs), one every $36 \mathrm{~m}$, with the inter-string distance of about $90 \mathrm{~m}$; while in ORCA the strings are $200 \mathrm{~m}$ high, with an inter-DOM spacing of only $9 \mathrm{~m}$ and an inter-line spacing of roughly $23 \mathrm{~m}$. Each 3" PMT, thanks to its construction design, permits to take into account and optimise the directional information sensibility of the KM3NeT DOMs. The first two lines of ARCA have been deployed in December 2015 and May 2016 respectively, the first string of ORCA has been deployed in September 2017.

\section{ARCA - Astroparticle Research with Cosmics in the Abyss}

The design of the KM3NeT DOMs allows to select atmospheric muons over the environmental background looking for local coincidences between the PMTs [2]. The decay of Potassium $40\left({ }^{40} \mathrm{~K}\right)$, dissolved in the sea water, in the vicinity of the DOM, causes the vast majority of coincident hits on 2 PMTs on the same DOM in a time window of $25 \mathrm{~ns}$. For the ${ }^{40} \mathrm{~K}$ individual events the distribution of the time difference $(\Delta t)$ is expected to follow a Gaussian distribution, whose the mean is the difference between the transit times of the involved PMTs, the width is related to the PMTs transit time spreads

\footnotetext{
ae-mail: paolo.fermani@roma1.infn.it
} 


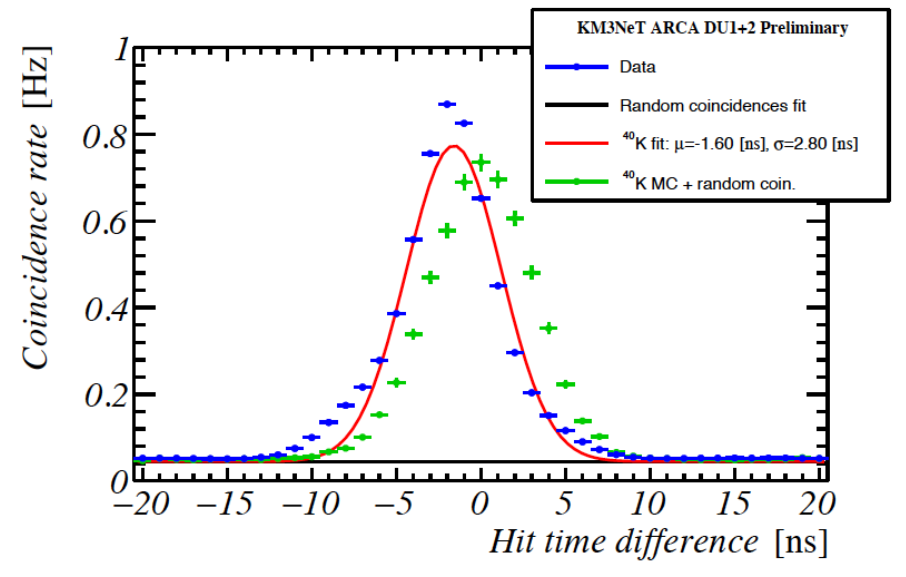

Figure 1: Coincidence rate vs $\Delta t$ for a pair of adjacent PMTs. Blue dots represent uncalibrated data. The red line is a Gaussian fit with mean equal to $-1.6 \mathrm{~ns}$ and standard deviation equal to $2.8 \mathrm{~ns}$. The green points show the expected hit time difference corrected with the addition of the background rate estimated from the tail of the data distribution (black solid line) [3].

and the integral under the curve proportional to the product of the PMT efficiencies [3]. The values to use for calibration of these quantities are derived from the comparison between the data and the Monte Carlo (MC) simulations of the ${ }^{40} \mathrm{~K}$ event distributions, as shown in figure 1 .

The rates of the multifold coincidences $(m)$ for the lowest and highest DOMs of the two strings of ARCA are shown in figure 2a together with the rates predicted by the MC simulations. No normalisation factor is applied to the MC simulations, showing an excellent absolute agreement between the data and the MC. At low $m$ the signal from ${ }^{40} \mathrm{~K}$ dominates the rates while the muon signature becomes dominant for $m \geq 8$.

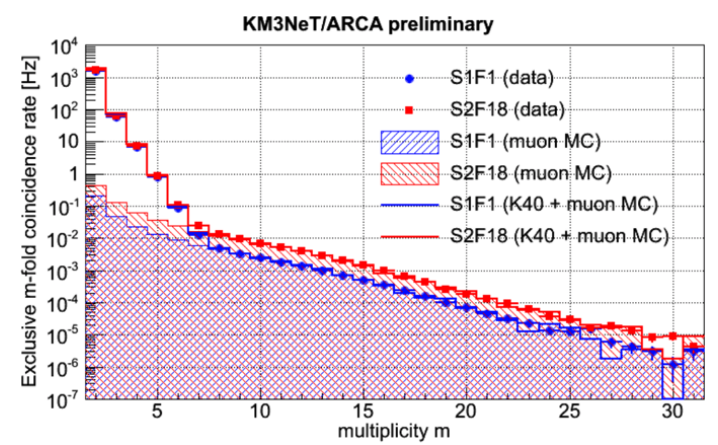

(a) Coincidence rate vs multiplicity.

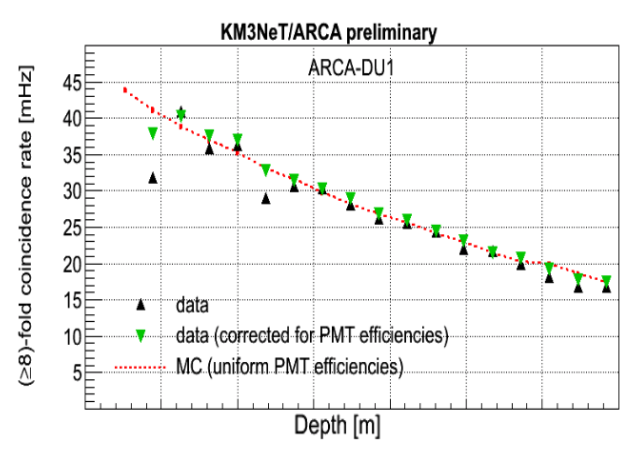

(b) $\geq 8$-fold coincidence rate vs sea depth.

Figure 2: (a) Coincidence rate vs multiplicity. Two DOMs are shown: floor 1 (the lowest floor) of ARCA-DU1 and floor 18 (the highest floor) of ARCA-DU2. Data are compared to with the expectations from MC simulations of ${ }^{40} \mathrm{~K}$ and atmospheric muons [4].

(b) $\geq 8$-fold coincidence rate $v$ s depth below the sea surface for ARCA string 1. The statistical error bars are too small to be visible [4].

The stability of the PMTs and the homogeneity of the detector performances have been verified through the study of the dependence of the multifold $(m \geq 8)$ coincidence rate on the sea depth [5]. In figure $2 \mathrm{~b}$ the red dashed line shows the coincidence rates as expected by MC simulations when 
uniform PMT efficiencies are considered neglecting the differences between individual PMTs. The black points show the uncorrected data and the green points show the data corrected according to the PMT efficiencies measured with in-situ calibration.

\section{ORCA - Oscillation Research with Cosmics in the Abyss}

The ORCA detector has a denser setup than the ARCA detector. This allows the detection of atmospheric neutrinos with an energy threshold $\mathrm{E}_{v} \approx 5-10 \mathrm{GeV}$. Dedicated analysis cuts are applied to the reconstructed tracks to select a clean neutrino candidate sample. With the available livetime of 82 days, 13 upgoing neutrino candidate events are observed, in good agreement with the MC prediction of $8.3 \pm 2.9$ (stat.) atmospheric neutrinos plus $1 \pm 1$ (stat.) events most likely due to bad reconstructed tracks. The zenith angle distribution for the selected tracks in the ORCA detector is in good agreement with $\mathrm{MC}$ predictions as shown in figure 3. Here the $\mathrm{MC}$ atmospheric muons are represented with blue crosses, the MC atmospheric neutrinos with red crosses and the data with black crosses [6].

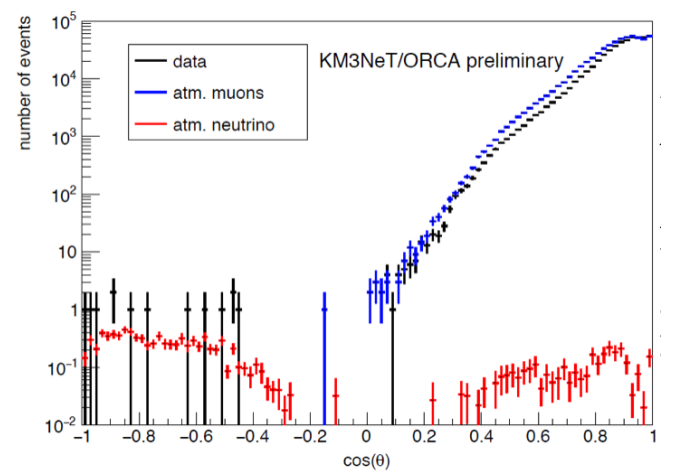

Figure 3: Reconstructed zenith angle distribution for the selected tracks in the ORCA detector [6].

\section{Conclusions}

These preliminary tests, both for ARCA and ORCA detectors, show the capabilities of the Cherenkov technique in sea water, verifiyng the KM3NeT technology and confirming the stability of the detector performances.

\section{References}

[1] S. Adrian-Martinez et al. (KM3NeT collaboration), J. Phys G 43, 084001 (2016)

[2] S. Adrian-Martinez et al. (KM3NeT collaboration), Eur. Phys. J. C 76, 54 (2016)

[3] K. Melis (on behalf of the KM3NeT collaboration), 35th ICRC, Busan, South Korea, PoS 301, 1059 (2017)

[4] M. Jongen (on behalf of the KM3NeT collaboration), 35th ICRC, Busan, South Korea, PoS 301, 1018 (2017)

[5] G. Ferrara, S. Biagi (on behalf of the KM3NeT collaboration), JPCS 1056, 012021 (2018)

[6] I. Di Palma, J. Hofestädt, D. Zaborov (on behalf of the KM3NeT collaboration), Results from the first ARCA and ORCA detector units, Poster presented at 28th Neutrino conference, Heidelberg, Germany (2018) 